\title{
Habilidades sociales y capacidad de manejo de conflictos interpersonales en docentes de un Instituto superior
}

\author{
Social skills and interpersonal conflict management skills of high school teachers \\ Habilidades sociais e habilidades de gerenciamento de conflitos interpessoais dos professores do \\ ensino médio
}

ARTícuLo dE INVESTIGACIÓN

Honorato Tomas Jaramillo Hernandez ${ }^{1}$

tomasito199729@gmail.com

https://orcid.org/0000-0002-8125-0233

\author{
Norka Inés Obregón Alzamora² \\ norkaobregon@gmail.com \\ https://orcid.org/0000-0002-7147-3548r
}

\section{Angel Hugo Pinedo Reyes ${ }^{3}$ \\ ahugopr2@gmail.com \\ https://orcid.org/0000-0003-0965-2921}

${ }^{1}$ Unidad de Gestión Educativa Local “Pomabamba”, Pomabamba, Perú

${ }^{2}$ Universidad Nacional Mayor de San Marcos, Lima, Perú

${ }^{3}$ Institución Educativa "Marcos Capristano Mori”, Sihuas, Perú

Recibido 6 de mayo 2021 | Arbitrado y aceptado 15 de junio 2021 | Publicado en 01 julio 2021

\section{RESUMEN}

Aborda el problema de las habilidades sociales y la capacidad de manejo de conflictos interpersonales, el cual se ha desarrollado teniendo como objetivo principal, identificar el grado de relación entre las habilidades sociales y el manejo de conflictos interpersonales; por lo mismo, adopta una investigación de tipo sustantiva con un diseño descriptivo correlacional, el cual se ha desarrollado en una población muestral de 45 docentes formadores del IESPP "Pomabamba". El procedimiento se expresa, en la aplicación de una escala de actitudes para medir el desarrollo de las habilidades sociales y otra para la capacidad de manejo de conflictos interpersonales, cuyos datos procesados, fueron sometidos a la prueba de Rho Spearman, obteniendo $r=0.558$, lo cual permite concluir que, cuanto más los docentes han desarrollado sus habilidades sociales, tienen mejores capacidades para manejar los conflictos interpersonales; determinándose finalmente que, existe fuerte relación entre las variables de estudio.

Palabras clave: Habilidades sociales; Manejo de conflictos; capacidades interpersonales
ABSTRACT

It addresses the problem of social skills and the ability to manage interpersonal conflicts, which has been developed with the main objective of identifying the degree of relationship between social skills and interpersonal conflict management; therefore, it adopts a substantive type of research with a descriptive correlational design, which has been developed in a sample population of 45 teachers trainers of the IESPP "Pomabamba". The procedure is expressed in the application of a scale of attitudes to measure the development of social skills and another for the ability to manage interpersonal conflicts, whose processed data were subjected to the Rho Spearman test, obtaining $\mathrm{r}=0.558$, which leads to the conclusion that the more teachers have developed their social skills, the better their ability to manage interpersonal conflicts; finally determining that there is a strong relationship between the variables under study.

Key words: Social skills; Conflict management; interpersonal skills

\section{RESUMO}

Ela aborda o problema das habilidades sociais e da capacidade de administrar conflitos interpessoais, que foi desenvolvida com o objetivo principal de identificar o grau de relacionamento entre as habilidades sociais e a administração de conflitos interpessoais; portanto, adota um tipo substantivo de pesquisa com um desenho descritivo correlacional, que foi desenvolvido em uma população de amostra de 45 professores formadores do IESPP "Pomabamba". O procedimento é expresso na aplicação de uma escala de atitudes para medir o desenvolvimento de habilidades sociais e outra para a capacidade de administrar conflitos interpessoais, cujos dados processados foram submetidos ao teste Rho Spearman, obtendo $r=0,558$, o que leva a concluir que quanto mais professores desenvolveram suas habilidades sociais, melhor será sua capacidade de administrar conflitos interpessoais; finalmente determinando que existe uma forte relação entre as variáveis em estudo.

Palavras-chave: Habilidades sociais; Gestão de conflitos; Habilidades interpessoais 


\section{INTRODUCCIÓN}

Las habilidades sociales yla capacidad de manejo de conflictos interpersonales, se desprenden de la necesidad de identificar el grado de relación entre las habilidades sociales avanzadas y la capacidad para preparar el escenario de mediación del conflicto interpersonal; además de las habilidades para manejar los sentimientos y la capacidad para caracterizar a las partes involucradas; también las habilidad para afrontar la agresión y la capacidad para generar alternativa de solución, así como las habilidades de planificación y la capacidad para finalizar el conflicto interpersonal.

En tal sentido y para desarrollar el presente estudio teóricamente sustentado, se tomó en cuenta lo señalado por Caballo (2007) quien afirma que las habilidades sociales son "Conjunto de conductas emitidas por un individuo en un contexto interpersonal, que expresa los sentimientos, actitudes, deseos, opiniones, o derechos de ese individuo de un modo adecuado a la situación, respetando esas conductas en los demás". (p. 6); por consiguiente Roca (2014) afirma que las habilidades sociales son "un conjunto de hábitos - en nuestras conductas, pero también en nuestros pensamientos y emociones - que nos permiten comunicarnos con los demás en forma eficaz, mantener relaciones interpersonales satisfactorias" (p. 5).

Díaz (2011) manifiesta que las habilidades sociales "han sido vistas como comportamientos que son instrumentales para resolver conflictos, situaciones o tareas sociales" (p. 24). De igual forma se toma en cuenta lo afirmado por Lineham (1984), quien asume que la habilidad social es una capacidad compleja para emitir conductas que optimicen la influencia interpersonal y la resistencia a la influencia social no deseada, mientras que al mismo tiempo optimiza las ganancias y minimiza las pérdidas en relación con las otras personas y mantiene la propia integridad.

En el entendido en las revisión de las literaturas consultadas en torno a las habilidades sociales y en concordancia con Ortego, López y Álvarez (2016), es posible concluir, que entre las definiciones existentes es posible distinguir los siguientes elementos y características: Son conductas que son socialmente aprendidas y aceptadas, las cuales son herramientas para interactuar con los demás con el propósito de lograr una meta propuesta. Así mismo tiene dentro de su constitución no solamente componentes afectivos, sino también elementos cognitivos, las mismas para ser asumidas como válidas y funcionales dependen del contexto, coyuntura, circunstancia y espacio donde se pretende aplicarlas.

En efecto, todo ello permite formular una concepción, en el sentido que las habilidades sociales vienen a ser conjunto de conductas y destrezas específicas que permiten al ser humano, relacionarse e interactuar con los demás demostrando mucho tino, para "leer" los sentimientos y expectativas del grupo; haciendo de esa interacción un espacio mutuamente beneficioso. En general, se hace referencia al conjunto de cogniciones, emociones y conductas que conllevan relacionarse y convivir con otras personas de forma satisfactoria y eficiente.

Por otro lado, en torno al manejo de conflictos interpersonales, se tuvo en cuenta los planteamientos teóricos de Hernández (2012), quien afirma que "los conflictos forman parte de la vida, son inherentes a las relaciones humanas y, sobre todo, son llamados de atención sobre alguna realidad que muy probablemente debe ser modificada para desactivarlos" (p. 69).

De los planteamientos realizados es posible considerar implica que, si los conflictos 
interpersonales, son abordados de una manera adecuada y positiva, pueden ser grandes oportunidades para aprender, madurar y mejorar en las relaciones que ejercemos como miembros de la sociedad; por lo tanto, las entidades educativas, deben preparar a sus estudiantes para ser capaces de solventar autogestivamente sus conflictos.

Los conflictos se asocian con faltas o transgresiones de normas, las mismas que son sancionadas por la autoridad del maestro o de la institución educativa; sin embargo, es importante reconocer que, no siempre es así; en otras ocasiones, estas controversias y desacuerdos surgen de la relación entre dos o más actores educativos, que pueden ser entre estudiante - estudiante, estudiante - docente, docente - directivos, como tal no siempre conlleva a una sanción, por el contrario, se procura generar condiciones para aclarar malentendidos y luego resolverlos.

Para tal efecto, son varias las habilidades que se ponen en juego, tales como la autorregulación personal, el control emocional, el diálogo, la escucha activa, la empatía, la creatividad, entre otras. Las habilidades, actitudes, comportamientos y valores que se sustentan en el respeto por sí mismo y por los demás, constituyen un elemento vital para superar situaciones conflictivas.

En ese entendido, el conflicto interpersonal es una situación en la que dos o más personas entran en oposición o desacuerdo de intereses o posiciones y la relación entre las partes puede terminar deteriorada en distintos grados; lo cual implica que la intensidad de las emociones y sentimientos, pueden provocar acercamiento o rechazo de las personas; que son por lo general inevitables, la gran mayoría pueden ser administrados y resueltos oportunamente.

Rojas (2010) manifiesta que alcanzar acuerdos armónicos es muy importante poner énfasis en los intereses y no en las posiciones de los involucrados; de ahí que se habla de la negociación basada en posiciones, conocida como "positional bargaining" por expertos de la Universidad de Harvard, en la cual, las partes asumen una postura, argumentan en su favor y se encierran dentro de ella; mientras más la defienden más se comprometen con una posición.

Por lo general, en este proceso, se suele partir de puntos de vista extremos y progresivamente se van otorgando concesiones, tan sólo cuando es necesario para proseguir con la negociación. En estos casos, son habituales los usos de tácticas dilatorias, amenazas de ruptura, inmovilismo. Este tipo de negociación mira el corto plazo, al poner énfasis en lo que se puede ganar de manera inmediata. En términos prácticos se produce una especie de competencia por obtener la mayor ganancia posible, sin que importen mucho las relaciones de vínculo que se puedan establecer en el futuro. Es lo que se conoce como ganar/perder. Lo que uno gana lo pierde el otro.

En síntesis, el manejo o resolución pacífica de conflictos interpersonales, es la intención y voluntad de las personas por resolver los conflictos, lo que implica, hacerlos visibles en la interacción interpersonal y/o grupal; considerar los intereses de las partes involucradas y la disposición a ceder para llegar a una salida que beneficie a los involucrados.

En base a lo expuesto se pudo formular con mayor precisión el problema de investigación basado en la siguiente interrogante ¿Cuál es la relación entre las habilidades sociales y la capacidad de manejo de los conflictos interpersonales en los docentes del Instituto de Educación Superior Pedagógico Público "Pomabamba”?; así como también responder con claridad a la pregunta ¿para qué investigar?, expresándolo en el objetivo general de la investigación que busca identificar el grado de 
relación de las habilidades sociales y la capacidad de manejo de los conflictos interpersonales en los docentes de la población muestral; ya que en la indicada institución formadora laboran maestros de distintas especialidades, quienes han recibido la formación inicial docente en institutos y/o universidades, algunos con pos grado, diplomados y especializaciones, con una carga laboral de 40 horas pedagógicas semanales; sin embargo no midieron tiempo y esfuerzo para formar parte de la unidad de análisis del presente estudio.

En efecto, se justifica por cuanto, en cada una de las aulas de las aulas del Instituto de Educación Superior Pedagógico Público "Pomabamba", donde los maestros prestan sus servicios profesionales como docentes de las diversas áreas y sub áreas curriculares, se observa a diario que, muchos de sus estudiantes presentan una serie de acciones y reacciones actitudinales que no concuerdan con las normas de convivencia establecida. Tal es así, no respetan los gustos y preferencias de los demás, mienten, agreden, evaden responsabilidades, marginan a sus compañeros, entre otros; los cuales, sino son tratados pertinente y oportunamente por el docente, pueden convertirse en verdaderos conflictos interpersonales, sobrepasándose inclusive las esferas institucionales. Esta situación, lógicamente exige del docente altos niveles de formación socioemocional, que se exprese en el tino y el sentido místico para manejar democrática y persuasivamente, los diversos conflictos que pudieran desencadenarse en la interacción cotidiana de los estudiantes e inclusive entre los propios docentes y trabajadores. Esta realidad empírica, pone en tapete el principio teórico que, para ser experto en el manejo de relaciones interpersonales es indispensable haber desarrollado previa y suficientemente las habilidades sociales, por lo que es una exigencia de primer nivel no solamente para los docentes quienes interactúan con adolescentes y jóvenes; sino también con niños.
Consecuentemente y así como la explicación efectuada por Robbins y Judge (2013), que en la negociación o manejo de conflictos influyen grandemente la capacidad para establecer relaciones interpersonales, en clara alusión a las habilidades sociales que son imprescindibles para afrontar inteligentemente exabruptos de esta naturaleza; a través del presente trabajo se proporciona valiosa información a los directivos, autoridades educativas y al mundo académico acerca de la situación socioemocional de los docentes, la cual sin duda es presupuesto indispensable para manejar apropiadamente los conflictos interpersonales.

En tal sentido, desde un punto de vista teórico, el estudio beneficiará a los investigadores y a los docentes en especial, para que puedan realizar una reflexión pedagógica y académica acerca del perfil socioemocional requerida para lidiar con los problemas de interacción de los estudiantes, haciendo que su intervención como mediador de conflictos interpersonales sea más efectiva y democrática. Aporta al conocimiento, identificando algunos factores decisivos en la adquisición de estrategias adecuadas para implantar una convivencia institucional armónica.

\section{MÉTODO}

Se realizó un estudio, descriptivo correlacional bajo la modalidad de trabajo de campo; siendo las unidades de análisis únicamente los 45 docentes formadores quienes, venían trabajando en condición de nombrados y contratados; de modo que el procedimiento muestral empleado fue no probabilístico con fines especiales.

De la población muestral descrita, se recopiló los datos, a través de la técnica proyectiva, en tanto para registrar los datos relacionados a ambas variables, se elaboró y aplicó escalas de actitudes de la siguiente manera: 
El primero, denominado escala de actitudes sobre habilidades sociales de los docentes, el cual es una adaptación del cuestionario de habilidades sociales de Goldstein (1980), que tiene por objetivo, identificar el nivel desarrollo de las habilidades sociales en los docentes de un Instituto de Educación Superior Pedagógico Público. Se aplicó en forma individual a través de un aplicativo en google drive, con una duración de 30 minutos aproximadamente, donde los investigados tuvieron la oportunidad de responder con: Me sucede escasas veces (1), me sucede pocas veces (2), me sucede bastantes veces (3) y me sucede siempre (4). Los ítems responden a las dimensiones de las habilidades sociales, tales como: Habilidades sociales avanzadas, habilidades para manejar los sentimientos, habilidades sociales alternativas a la agresión y las habilidades de planificación.

$\mathrm{Si}$ bien es cierto, que el cuestionario de habilidades sociales fue diseñada y elaborada por Arnold Goldstein en Nueva York, Estados Unidos en 1978, el mismo que fue traducida inicialmente por Rosa Vásquez en 1983, posteriormente la versión final fue traducida, adaptada, validada y estandarizada en el medio por Rojas en (19941995), en esta ocasión también se ha efectuado una adaptación al nivel profesional de los miembros de la muestra de estudio.

En lo que respecta, a la confiabilidad de este instrumento, hace referencia, según Chávez (2001) al grado con el que se obtienen resultados similares en distintas aplicaciones. En efecto, sobre este particular se realizó el cálculo del coeficiente de Alfa de Cronbach mediante una prueba piloto realizada a 10 docentes quienes no formaron de la muestra de estudio, obteniendo un valor de 0,966, lo cual significa que existe alta confiabilidad en el instrumento.

El segundo instrumento, denominado, escala de actitudes de la capacidad de manejo de conflictos interpersonales, es de elaboración propia, con el objetivo de medir la capacidad que tienen los docentes de un Instituto de Educación Suprior Pedagógico Público, para manejar democráticamente los conflictos interpersonales que pudieran ocurrir en el interior del aula, ya sea en las relaciones entre estudiantes o estudiantes y docentes. Los integrantes de la población y muestra de estudio a la vez, respondieron una escala de Likert, en un formulario elaborado y proporcionado en línea a través de google drive, por un espacio de 30 minutos como máximo, cuyo conteo ya se tenía en tiempo real. Los valores que evalúa son: Preparación del escenario de la mediación, caracterización de las partes y las cuestiones en controversia, búsqueda de alternativas de solución y la finalización de la mediación del conflicto; de manera que, para cada una de ellas, se formuló 05 indicadores, con cuatro categorías cada una, tales como: Escasas veces (1), a veces (2), muchas veces (3) y siempre (4), los cuales al ser procesados se obtuvo como máximo un calificativo vigesimal.

Tomando como marco de referencia a lo planteado por Trechera (2004) respecto a los procesos de mediación eficaz de un conflicto interpersonal, se formuló los ítems de la escala de actitudes, con 04 categorías cada una; los cuales tuvieron que pasar por criterios de validez y confiabilidad.

Para la versión original del instrumento, se estableció la confiabilidad por consistencia interna aplicando el coeficiente Alpha de Cronbach, obteniéndose el valor 0,073 , el cual también significa que, existe alta confiabilidad del instrumento.

\section{RESULTADOS Y DISCUSIÓN}

El resultado analítico y por dimensiones son expresadas en las siguientes tablas: 
Tabla 1. Relación entre las habilidades sociales avanzadas y la capacidad para preparar el escenario de mediación del conflicto interpersonal, en los docentes del IESPP “Pomabamba”.

\begin{tabular}{|c|c|c|c|c|}
\hline & & \multicolumn{2}{|c|}{ Habilidades sociales avanzadas } & $\begin{array}{l}\text { Capacidad para preparar el } \\
\text { escenario de mediación de }\end{array}$ \\
\hline \multirow[t]{6}{*}{ Rho de Spearman } & \multirow[t]{3}{*}{$\begin{array}{l}\text { Habilidades } \\
\text { sociales avanzadas }\end{array}$} & Coeficiente de correlación & 1.000 &, $404^{\star *}$ \\
\hline & & Sig. (bilateral) & & .006 \\
\hline & & $\mathrm{N}$ & 45 & 45 \\
\hline & \multirow{3}{*}{$\begin{array}{l}\text { Capacidad } \\
\text { para preparar } \\
\text { el escenario de } \\
\text { mediación de } \\
\text { conflictos }\end{array}$} & Coeficiente de correlación &, $404^{* *}$ & 1.000 \\
\hline & & Sig. (bilateral) & .006 & \\
\hline & & $\mathrm{N}$ & 1.000 &, $404^{\star *}$ \\
\hline
\end{tabular}

**. La correlación es significativa en el nivel 0,01 (2 colas).

\section{Criterio de decisión}

Si $\mathrm{p}<0.05$ se rechaza la $\mathrm{H}_{0}$ y se acepta la $\mathrm{H}_{\mathrm{i}}$

Si $\mathrm{p} \geq 0.05$ se acepta la $\mathrm{H}_{0}$ y se rechaza la $\mathrm{H}_{\mathrm{i}}$

\section{Decisión e interpretación}

Como el valor $\mathrm{p}=006$, el cual es inferior al valor de significancia establecida por el investigador, que es $\alpha=0,05$; se rechaza la hipótesis nula $\left(\mathrm{H}_{0}\right)$ y se acepta la hipótesis del investigador $\left(\mathrm{H}_{\mathrm{i}}\right)$; e inclusive siendo $r=0.404$, se puede concluir que, existe fuerte relación entre las habilidades sociales avanzadas y la capacidad para preparar el escenario para la mediación de conflictos interpersonales; es decir, cuanto más los docentes hayan desarrollado sus habilidades sociales avanzadas tendrían mejores capacidades para preparar un escenario apropiado para mediar los conflictos interpersonales y viceversa.

Tabla 2. Relación entre las habilidades para manejar los sentimientos y la capacidad para caracterizar a los protagonistas del conflicto interpersonal, en los docentes del IESPP "Pomabamba".

\begin{tabular}{|c|c|c|c|c|}
\hline & & \multicolumn{2}{|c|}{$\begin{array}{c}\text { Habilidades sociales para manejar los } \\
\text { sentimientos }\end{array}$} & $\begin{array}{c}\text { Capacidad para caracterizar a } \\
\text { los protagonistas del conflicto } \\
\text { interpersonal }\end{array}$ \\
\hline \multirow[t]{3}{*}{ Rho de Spearman } & \multirow[t]{2}{*}{$\begin{array}{l}\text { Habilidades } \\
\text { sociales para } \\
\text { manejar los } \\
\text { sentimientos }\end{array}$} & Sig. (bilateral) & 1.000 &, $388^{\star *}$ \\
\hline & & $\mathrm{N}$ & 45 & 45 \\
\hline & $\begin{array}{l}\text { Capacidad para } \\
\text { caracterizar a los } \\
\text { protagonistas } \\
\text { del conflicto } \\
\text { interpersonal }\end{array}$ & $\begin{array}{l}\text { Coeficiente de correlación } \\
\text { Sig. (bilateral) }\end{array}$ & $\begin{array}{l}.388^{\star *} \\
.008\end{array}$ & 1.000 \\
\hline
\end{tabular}

**. La correlación es significativa en el nivel 0,01 (2 colas). 


\section{Criterio de decisión}

Si $\mathrm{p}<0.05$ se rechaza la $\mathrm{H}_{0}$ y se acepta la $\mathrm{H}_{\mathrm{i}}$ Si $\mathrm{p} \geq 0.05$ se acepta la $\mathrm{H}_{0}$ y se rechaza la $\mathrm{H}_{\mathrm{i}}$

\section{Decisión e interpretación}

Siendo el valor $\mathrm{p}=008$, inferior al valor de significancia establecida que es $\alpha=0,05$; se rechaza la hipótesis nula $\left(\mathrm{H}_{0}\right)$ y se acepta la hipótesis del investigador $\left(\mathrm{H}_{\mathrm{i}}\right)$; e inclusive como $\mathrm{r}=0.338$, se puede concluir que, existe fuerte relación entre las habilidades sociales para manejar los sentimientos y la capacidad para caracterizar a los protagonistas de los conflictos interpersonales; es decir, cuanto más los docentes hayan desarrollado sus habilidades sociales para manejar los sentimientos tendrían mejores capacidades para caracterizar a los protagonistas de los conflictos interpersonales y viceversa.

Tabla 3. Relación entre las habilidades alternativas a la agresión y la capacidad para generar las alternativas de solución del conflicto interpersonal, en los docentes del IESPP "Pomabamba".

\begin{tabular}{|c|c|c|c|c|}
\hline \multirow[b]{2}{*}{ Rho de Spearman } & \multirow{3}{*}{$\begin{array}{l}\text { Habilidades } \\
\text { alternativas a la } \\
\text { agresión }\end{array}$} & \multicolumn{2}{|c|}{ Habilidades alternativas a la agresión } & \multirow{2}{*}{$\begin{array}{c}\text { Capacidad para generar las } \\
\text { alternativas de solución del } \\
\text { conflicto interpersonal }\end{array}$} \\
\hline & & Coeficiente de correlación & 1.000 & \\
\hline & & Sig. (bilateral) & & .000 \\
\hline & & $\mathrm{N}$ & 45 & 45 \\
\hline & \multirow{3}{*}{$\begin{array}{l}\text { Capacidad } \\
\text { para generar } \\
\text { las alternativas } \\
\text { de solución } \\
\text { del conflicto } \\
\text { interpersonal }\end{array}$} & Coeficiente de correlación &, $566^{* *}$ & 1.000 \\
\hline & & Sig. (bilateral) & .000 & \\
\hline & & $\mathrm{N}$ & 45 & 45 \\
\hline
\end{tabular}

**. La correlación es significativa en el nivel 0,01 (2 colas).

\section{Criterio de decisión}

Si $\mathrm{p}<0.05$ se rechaza la $\mathrm{H}_{0}$ y se acepta la $\mathrm{H}_{\mathrm{i}}$

Si $\mathrm{p} \geq 0.05$ se acepta la $\mathrm{H}_{0}$ y se rechaza la $\mathrm{H}_{\mathrm{i}}$

\section{Decisión y conclusión}

Como el valor $\mathrm{p}=000$, es inferior al valor de significancia establecida que es $\alpha=0,05$; entonces se rechaza la hipótesis nula $\left(\mathrm{H}_{0}\right)$ y se acepta la hipótesis del investigador $\left(\mathrm{H}_{\mathrm{i}}\right)$; e inclusive siendo $\mathrm{r}$ $=0.566$, se puede concluir que, existe fuerte relación entrelas habilidades sociales alternativas a la agresión y la capacidad para plantear alternativas de solución a los conflictos interpersonales; es decir, cuanto más los docentes hayan desarrollado sus habilidades sociales alternativas a la agresión tendrían mejores capacidades para proponer alternativas más viables a los conflictos interpersonales y viceversa. 
Tabla 4. Relación entre las habilidades de planificación y la capacidad de finalización de la mediación del conflicto interpersonal en los docentes del IESPP "Pomabamba".

\begin{tabular}{|c|c|c|c|c|}
\hline & & \multicolumn{2}{|c|}{ Habilidades de planificación } & $\begin{array}{l}\text { Capacidad de finalización } \\
\text { de la mediación del conflicto }\end{array}$ \\
\hline \multirow[t]{6}{*}{ Rho de Spearman } & \multirow[t]{3}{*}{$\begin{array}{l}\text { Habilidades de } \\
\text { planificación }\end{array}$} & Coeficiente de correlación & 1.000 &, $563^{\star *}$ \\
\hline & & Sig. (bilateral) & & .000 \\
\hline & & $\mathrm{N}$ & 45 & 45 \\
\hline & \multirow{3}{*}{$\begin{array}{l}\text { Capacidad de } \\
\text { finalización de } \\
\text { la mediación } \\
\text { del conflicto } \\
\text { interpersonal }\end{array}$} & Coeficiente de correlación &, $563^{\star *}$ & 1.000 \\
\hline & & Sig. (bilateral) & .000 & \\
\hline & & $\mathrm{N}$ & 45 & 45 \\
\hline
\end{tabular}

**. La correlación es significativa en el nivel 0,01 (2 colas).

\section{Criterio de decisión}

Si $\mathrm{p}<0.05$ se rechaza la $\mathrm{H}_{0}$ y se acepta la $\mathrm{H}_{\mathrm{i}}$

Si $\mathrm{p} \geq 0.05$ se acepta la $\mathrm{H}_{0}$ y se rechaza la $\mathrm{H}_{\mathrm{i}}$

\section{Decisión e interpretación}

Siendo $\mathrm{p}=000$, muy inferior al valor de significancia establecida que es $\alpha=0,05$; entonces se rechaza la hipótesis nula $\left(\mathrm{H}_{0}\right)$ y se acepta la hipótesis del investigador $\left(\mathrm{H}_{\mathrm{i}}\right)$; e inclusive como $\mathrm{r}=$ 0.563 , se puede concluir que, existe fuerte relación entre las habilidades sociales de planificación y la capacidad para finalizar democráticamente a los conflictos interpersonales; es decir, cuanto más los docentes hayan desarrollado sus habilidades sociales de planificación tendrían mejores capacidades para finalizar adecuadamente a los conflictos interpersonales y viceversa.

Tabla 5. Niveles relación entre las habilidades sociales y la capacidad de manejo los conflictos interpersonales en los docentes del Instituto de Educación Superior Pedagógico Público “Pomabamba.

\begin{tabular}{|c|c|c|c|c|}
\hline & & \multicolumn{2}{|c|}{ Habilidades sociales } & $\begin{array}{l}\text { Capacidad de manejo de } \\
\text { conflictos interpersonales }\end{array}$ \\
\hline \multirow[t]{6}{*}{ Rho de Spearman } & \multirow[t]{3}{*}{$\begin{array}{l}\text { Habilidades } \\
\text { sociales }\end{array}$} & Coeficiente de correlación & 1.000 &, $558^{\star *}$ \\
\hline & & Sig. (bilateral) & & .000 \\
\hline & & $\mathrm{N}$ & 45 & 45 \\
\hline & \multirow{3}{*}{$\begin{array}{l}\text { Capacidad } \\
\text { de manejo } \\
\text { de conflictos } \\
\text { interpersonales }\end{array}$} & Coeficiente de correlación &, $558^{\star *}$ & 1.000 \\
\hline & & Sig. (bilateral) & .000 & \\
\hline & & $\mathrm{N}$ & 45 & 45 \\
\hline
\end{tabular}

**. La correlación es significativa en el nivel 0,01 (2 colas). 


\section{Criterio de decisión}

Si $\mathrm{p}<0.05$ se rechaza la $\mathrm{H} 0$ y se acepta la $\mathrm{Hi}$

Si $\mathrm{p} \geq 0.05$ se acepta la $\mathrm{H} 0$ y se rechaza la Hi

\section{Decisión e interpretación}

Como el valor $\mathrm{p}=000$ es muy inferior al valor de significancia establecida que es $\alpha=0,05$; se rechaza la hipótesis general nula (H0) y se acepta la hipótesis general del investigador (Hi); e inclusive siendo $\mathrm{r}=0.558$, se puede concluir que, existe fuerte relación entre las habilidades sociales y la capacidad de manejo de conflictos interpersonales; es decir, cuanto más los docentes hayan desarrollado sus habilidades sociales tendrían mejores capacidades para manejar los conflictos interpersonales $y$ viceversa.

\section{Discusión}

En atención directa al objetivo general, se pudo identificar que, entre las habilidades sociales y la capacidad de manejo de conflictos interpersonales en los docentes del IESPP "Pomabamba" se encuentran fuertementerelacionadas; lo cual implica que cuanto más han desarrollado los docentes sus habilidades sociales, demuestran mejores y mayores capacidades para afrontar democráticamente los conflictos dentro y fuera del aula.

Este resultado concuerda ampliamente con lo descubierto por Lucicleide de Souza (2011), quien en su tesis "Competencias emocionales y resolución de conflictos interpersonales en el aula”, señala que, los profesores promueven en las aulas escasas actividades para desarrollar las competencias emocionales de los estudiantes, lo cual es considerada como un error, toda vez que el apoyo emocional del profesorado en el proceso de aprendizaje es esencial; sin embargo, marca distancia entre lo investigado por Bolaños (2015), quien en su tesis "Relaciones interpersonales docentes y manejo de conflictos administrativos educativos", concluye que, no es evidente relación entre las relaciones interpersonales de los docentes y el manejo de conflictos administrativos educativos en el distrito 090105. Todo lo afirmado recibe un sustento teórico en Díaz (2011) quien afirma que, las habilidades sociales son consideradas como comportamientos instrumentales para resolver conflictos, situaciones o tareas sociales.

En consecuencia y en atención a Rodríguez (2017), quien en su tesis "La mediación en los conflictos interpersonales entre directivos y maestros de las escuelas secundarias técnicas del municipio de Monterrey Nuevo León", señala que, las prácticas regenerativas constituyen, la vía de solución de conflictos más idónea de conflictos, porque toma en cuenta el control y la gestión de emociones como son, el odio, la angustia, resentimiento y hasta el perdón, los cuales son considerados cruciales e indispensables, por lo que, sobre todo los maestros y los futuros maestros, deben desarrollar y ser partes de una serie de talleres y cursos de habilidades sociales, por cuanto en las aulas de hoy más que nunca se requiere a maestros que sean expertos en la resolución de problemas. Todo ello, en contraposición a lo señalado por Bolaños (2015), quien afirma lo contrario.

\section{CONCLUSIONES}

En atención al objetivo general, se demostró que, entre las habilidades sociales y la capacidad para el manejo de conflictos interpersonales en los docentes del IESPP "Pomabamba", existe fuerte relación; lo cual, implica que, cuanto más los docentes han desarrollado sus habilidades sociales avanzadas, habilidades para manejar los sentimientos, para afrontar la agresión y las referidas a la planificación, tendrían mejores capacidades no solamente para preparar el escenario de la mediación, sino también para caracterizar a las partes involucradas, generar alternativas de solución con la intervención de 
los propios protagonistas y finalizar el proceso de mediación logrando que no resulten ganadores ni perdedores.

De manera analítica, estableciendo la relación entre las dimensiones de la variable X e Y, se ha arribado a las siguientes conclusiones específicas que responden a los objetivos específicos:

Entre las habilidades sociales avanzadas y la capacidad para preparar el escenario de la mediación por parte de los docentes; existe fuerte relación; es decir, los docentes quienes tienen dentro de su práctica pedagógica, la costumbre de iniciar el proceso de mediación del conflicto, preparando o fijando un ambiente o escenario libre de todo tipo de presión o distracción, son aquellos que saben pedir ayuda, así como también están en condiciones de brindar y seguir instrucciones $y$ discutir y convencer a los demás.

Así mismo, las habilidades sociales para manejar los sentimientos y la capacidad para caracterizar a las partes y las cuestiones en controversia, experimentan relación significativa; lo cual significa que, cuanto más un docente se encuentra en condiciones para reconocer las emociones, angustias y miedos propios y ajenos, estaría en mejores posibilidades para "leer" los intereses y expectativas, así como el carácter y temperamento de los protagonistas del conflicto.

También se identifica que, entre las habilidades sociales alternativas a la agresión y la capacidad para generar alternativas de solución; existe fuerte relación; lo cual implica que, si los docentes pueden y saben controlar sus emociones, evitan las peleas de manera inteligente, responden con tino a las amenazas y se defienden sin agredir a los demás; estarían preparados para acoger democráticamente a las propuestas, evaluarlas objetivamente $y$ establecer acuerdos y detalles coherentes frente a los conflictos interpersonales.

Finalmente, existe una relación significativa entre las habilidades sociales de planificación y la capacidad para finalizar la mediación del conflicto interpersonal; lo cual implica que, los docentes quienes tiene entre sus hábitos tomar iniciativas, contar con objetivos claros, priorizar la solución de problemas; estarían en mejores condiciones para resolver los conflictos en primera instancia, establecer acuerdos formales y fomentar acciones de control de cumplimiento de los mismos.

\section{REFERENCIAS}

Bolaños, J. (2015). Relaciones interpersonales docentes y manejo de conflictos administrativos educativos. Tesis para optar el título de pedagogo con orientación en administración y evaluación educativas en el grado académico de licenciado, en la Universidad Rafael Landívar. Recuperado de http://recursosbiblio.url.edu.gt/ tesiseortiz/2015/05/09/Bolanos-Jose.pdf

Caballo, V. (2007). Manual de entrenamiento $y$ evaluación de habilidades sociales. Madrid: Siglo XXI

Díaz Rodríguez, M., Puertas Cristóbal, E., y Medrano Moncada, Y. (2011). Habilidades de comunicación para la vida cotidiana

Goldstein, A. (1980). Lista de Chequeo de Habilidades Sociales (LCHS). Madrid: Projective Way

Hernández, G. (2012). Manual de gestión y resolución de conflictos. México: Indesol

Lineham, M. (1984). Interpersonal effectiviness in assertive situations. EA Bleechman (Com), Nueva York

Lucicleide de Souza (2011). Competencias emocionales y resolución de conflictos interpersonales en el aula. Tesis para optar el grado académico de Doctor, en la Universidad Autónoma de Barcelona. Recuperado de https:// www.eumed.net/tesis-doctorales/2012/lsb/ ficha.htm

Ortego, M., López, S. y Álvarez, M. (2016). Ciencias psicosociales I. Santander: Universidad de Cantabria

Robbins y Judge (2013). Comportamiento Organizacional. México: Pearson 
Roca, E. (2014). Cómo mejorar tus habilidades sociales. Valencia: ACDE

Rodríguez, D. (2017). La mediación en los conflictos interpersonales entre directivos y maestros de las escuelas secundarias técnicas del Municipio de Monterrey Nuevo León". Tesis para optar el grado académico de doctor en métodos alternos en solución de conflictos, en la Universidad Autónoma de Nuevo León. Recuperado de http://eprints.uanl.mx/13941/1/1080216536. pdf

Rojas, C. (2010). Guía didáctica para docentes: El Conflicto como una oportunidad. Inclusión y Equidad Consultora. Chile 